\title{
Depression, anxiety and stress among dental students during COVID-19 pandemic and distance learning
}

Francisco Ivison Rodrigues Limeira*; Poliana Farias da Cruz**; Amanda Ribeiro da Costa Andrade**; Armando Baia Guiomarino Neto***; Diandra Costa Arantes****

\author{
* Professor, School of Dentistry, Faculdade Presidente \\ Antônio Carlos \\ ** Undergraduate student, School of Dentistry, Faculdade \\ Presidente Antônio Carlos \\ *** Undergraduate student, School of Dentistry, Universidade \\ Federal do Pará \\ **** Adjunct Professor, School of Dentistry, Universidade \\ Federal do Pará
}

Received: 03/28/2021. Approved: 01/03/2022.

\begin{abstract}
This study aimed to measure the levels of depression, anxiety and stress among dental students during the COVID-19 pandemic and distance learning. This cross-sectional questionnaire-based survey was conducted at a Brazilian school of Dentistry, between July and August, 2020. The participants were surveyed using the adapted and validated version of Depression, Anxiety, and Stress Scale (DASS21) questionnaire. The Fisher's exact test and bivariate logistic regression analysis were performed to analyze the data, using SPSS software. A total of 120 dental students (response rate of $87.60 \%$ ) participated in the study; $75.8 \%$ of them were female and the mean age was 23.35 years old $( \pm 6.07)$. Most students were single (85\%), studied full time (68.3\%) and had no employment $(70.8 \%)$. Some level of depression, anxiety and stress was observed in $64.2 \%, 67.5 \%$ and $61.7 \%$ of the students, respectively. The severity of the depression, the anxiety and the stress was significantly associated (p $<0.05$ ) with the student's gender, fear of contracting COVID-19 and with the condition of having a family member who has already had COVID-19. Female students, students who were fear of contracting COVID-19 and who had a familiar or a friend diagnosed with COVID-19 presented more chances of developing a high level of depression, anxiety or stress. We concluded that dental students had high levels of depression, anxiety and stress during the COVID-19 pandemic and distance learning. This study suggests that the mental health of dental students should be carefully monitored during the COVID-19 pandemic.
\end{abstract}

Descriptors: Coronavirus Infections. Education, Dental. Mental Health.

\section{INTRODUCTION}

China was the first country that identified the new coronavirus disease (COVID-19). The World Health Organization (WHO) declared the COVID-19 an international public health emergency on January 30 2020, and a pandemic on March 11, 20201. Brazil was the first South American country to report a confirmed case of COVID-19, on February 26, 2020². Since then, the country has presented a complex 
epidemiological scenario, with regional differences $^{3}$. The fast evolution of the situation has drastically altered people's lives, as well as multiple aspects of the global, public and private, economy ${ }^{4}$.

Together with the strong person-to-person transmission capability of the virus, the tension that COVID-19 poses on public health and the implemented restrictions may have detrimental effects on mental health ${ }^{5}$. According to a systematic review, the COVID-19 pandemic is associated with significant levels of psychological distress that, in many cases, would meet the threshold for clinical relevance. Thus, mitigating the hazardous effects of COVID-19 on mental health should be an international public health priority ${ }^{4}$.

Although the overall impact on education and mental health of the university environment is still little known, it is expected to be very considerable ${ }^{6,7}$. Considering the usual high incidence of emotional disorders in university students, it can be expected that the current situation may cause a notable impact on this population $^{8,9,10}$. Recent studies show that the current COVID-19 pandemic is making a significant negative impact on the mental health of university students, with a high prevalence of posttraumatic, anxiety and depressive symptoms $^{11,12,13}$. Students who exhibit greater academic and life difficulties may be particularly vulnerable to higher mental health distress. Furthermore, the timeline of the pandemic is uncertain further impacting students' academics, lives and mental health ${ }^{11}$.

Dental schools are reported to be highly demanding and stressful learning environments $^{14}$. Studies have shown that studying dentistry can be extremely stressful for students who need to acquire diverse proficiencies such as theoretical knowledge, clinical skills, and interpersonal communication skills ${ }^{15,16}$. Stress might result in various mental and physical problems leading to exhaustion as well as physical and mental illnesses ${ }^{17}$. Furthermore, it results in diminished efficiency in learning ${ }^{18}$. In March 2020, the COVID-19 pandemic necessitated the immediate closure of in-person dental education to comply with social regulations ${ }^{19}$. Dental schools faced the challenge of effectively engaging students over virtual learning platforms ${ }^{20}$. The success of distance learning depends on the attitudes and interactive teaching styles of the faculty, as well as on the experience and attitudes of students regard to technology ${ }^{21}$. This new situation leads to an increase in the degree of anxiety among students who must adjust to new methods of educational instruction and are fearful for their safety and health ${ }^{19}$.

There are still no studies that have assessed the impact of the COVID-19 pandemic on the mental health of university dental students in Brazil, although the current context of dental education may favor the incidence of emotional disorders. Thus, this study aimed to measure the levels of depression, anxiety and stress among dental students during the COVID-19 pandemic and distance learning.

\section{METHODS}

This cross-sectional questionnaire-based survey was conducted at the School of Dentistry, Faculty President Antônio Carlos, Brazil. The protocol of this study was approved by the local ethics committee (\#4.114.334), according to the resolution no. 466/12 of the National Health Council. Informed written consent was obtained from all individuals participating in the study. The SURGE reporting guideline was consulted ${ }^{22}$. The school of dentistry where this study was conducted started in 2019. With the advancement of the COVID-19 pandemic, faceto-face classes were suspended at this Faculty on 
March 17, 2020. After this date, classes took place through a virtual learning platform, with synchronous classes. This study was carried out at the end of the first academic semester developed with this methodology, between July and August of 2020.

In July 2020, there were classes in the first and second periods and a total of 137 students. The sample size was calculated using an online power and sample size calculator (http://powerandsamplesize.com/) considering a 95\% significance level, $5 \%$ acceptable margin of error and $50 \%$ prevalence rate. The sample was then increased $10 \%$ to compensate for possible refusal rate. Thus, the minimum final sample comprised 113 students. All undergraduate dental students were eligible to participate. However, those with age under 18 years and that receiving any psychological management (cognitive or behavioral therapy, medication, or a combination; whose responses could be biased due to the effect of treatment on their psychological status) were excluded.

A self-administered questionnaire was developed with four sections and hosted online on the Google Forms platform (Google Inc., Menlo Park, CA, USA). For all dental students, invitations to participate were sent by e-mail. The e-mail contained a brief statement that included the study objective, the average response time and a website link to the questionnaire. The first section of the questionnaire included questions related to socio-demographic characteristics such as gender, age, family income, employment and marital status. The second section involved questions about the COVID-19 pandemic such as a positive diagnosis for COVID-19, fear of contracting COVID-19, the existence of infected family or friends and the practice of social detachment. The third section contained issues related to distance learning such as availability of the internet and digital device, good computer skills and degree of satisfaction. The fourth section of the questionnaire contained the items from of the short-form version of the Depression, Anxiety and Stress Scale (DASS-21).

The DASS questionnaire was first developed by Lovibond and Lovibond (1995) ${ }^{23}$. The DASS-21 questionnaire is a short-form version of the DASS that measures the negative emotional states of depression, anxiety, and stress by the sum of the seven corresponding questions $^{24}$. The questionnaire had twenty one items which were rated on a 4-point Likert scale (from 0 [did not apply to me at all] to 3 [applied to me very much or most of the time]). The higher the grade, the higher the levels of stress. The final score for stress, anxiety or depression was obtained by doubling the initial sum of the scales of the seven corresponding questions because the DASS-21 (21 items) is a short-form version of the DASS (forty two items). The following cut-off scores are used for each subscale: Depression: normal 0-9, mild 10-13, moderate 14-20, severe 21-27 and extremely severe $\geq 28$; Anxiety: normal 0-7, mild 4-5, moderate 8-9, severe 10-14 and extremely severe $\geq 20$; Stress: normal 0-14, mild 15-18, moderate 19-25, severe 26-33 and extremely severe $\geq 34 .{ }^{24}$ The DASS-21 demonstrated good to excellent internal consistency ${ }^{25}$, adequate reliability and construct validity ${ }^{26}$. An adapted and validated version of this questionnaire for use in the Portuguese from Brazil was used in this study $^{27}$.

The Fisher's exact test was used to analyze possible differences among the severity of the depression, anxiety and stress, according to socio-demographic characteristics, and questions about the COVID-19 pandemic and distance learning. Bivariate logistic regression analysis was performed to explore the association between the severity of depression, anxiety and stress with these characteristics and questions. 
For this analysis, the severity of the depression, the anxiety and the stress were divided into two categories: 1) Low, including "normal", "mild" and "moderate" levels; and 2) High, including "severe" and "extremely severe" levels. All analyses considered a $5 \%$ level of significance and were performed using the statistical software SPSS Statistics (Version 23.0. Armonk, NY: IBM Corp).

\section{RESULTS}

Among the 137 enrolled dentistry students, 120 accepted to participate in the study, which represents a response rate of $87.60 \%$, above the number defined by the sample calculation. These, $75.8 \%$ were female and $24.2 \%$ were male, with an average age of 23.35 years $( \pm 6.07)$. About $44 \%$ have monthly family income between three and four minimum wages (Brazilian minimum wage equals 198 USD, considering the quotation of July 2020). Most students were single (85\%), studied full time $(68.3 \%)$ and had no employment (70.8\%). Almost all (97.5\%) students said they had an available internet connection and $86.7 \%$ and confirmed having good knowledge about internet tools. However, less than half $(44.2 \%)$ of students were satisfied with distance learning. Regarding the COVID-19 pandemic, $10.8 \%$ said they had been diagnosed with the COVID-19, $80 \%$ were fear of contracting COVID-19, $57.5 \%$ and $61.6 \%$ had a family member and friend diagnosed with the COVI-19, respectively, and $83.3 \%$ were in social detachment (table 1).

Some levels of depression, anxiety and stress were observed in $64.2 \%, 67.5 \%$ and $61.7 \%$ of the students, respectively. The mean total scores for the individuals were $1.82( \pm 1.60)$ for depression, $2.26( \pm 1.79)$ for anxiety and $1.70( \pm$ 1.64) for stress. Alarmingly, severe and extremely severe scores for depression, anxiety, and stress were reported in $38.3 \%, 50.8 \%$ and
$35.5 \%$ of students, respectively (table 1).

The severity of the depression, anxiety and stress was significantly associated $(p<0.05)$ with the student's gender, the fear of contracting COVID-19 and with the condition of having a family member who has already had COVID-19. The severity of the depression and stress presented a significant difference between students who were or were not satisfied with remote teaching. There was also an association between the severity of the depression and anxiety with the knowledge about internet tools $(\mathrm{p}=0.02)$ and with the condition of having a friend who has already had COVID-19 $(\mathrm{p}=0.00)$ (table 1).

Bivariate logistic regression analysis showed that students who were afraid with COVID-19 (OR = 20.29), who had a familiar $(\mathrm{OR}=3.15)$, or a friend $(\mathrm{OR}=5.01)$ with COVID-19 presented more chances of developing a high level of depression $(p=0.00)$. As well as, students who were afraid of COVID$19(\mathrm{OR}=5.32)$, who had a familiar $(\mathrm{OR}=6.68)$, or a friend $(\mathrm{OR}=5.55)$ with COVID-19 presented more chances of developing a high level of anxiety. Female students had more chances to develop a high level of anxiety $(\mathrm{OR}=$ $2.42)$ and stress $(O R=10.58)$ than male students. Students who were fear of contracting COVID19 had much more chances to present of a high level of stress too $(\mathrm{OR}=17.14)$ (table 2$)$.

\section{DISCUSSION}

Until the moment, this study is the first to evaluate the impact of the COVID-19 pandemic on the mental health of university dental students in Brazil. This cross-sectional questionnairebased survey demonstrated overall elevated levels of depression, anxiety, and stress among dental students during the COVID-19 pandemic and distance learning. Moreover, female students, students who were fear of contracting 
Depression, anxiety and stress among dental students during COVID-19 pandemic and distance learning

Table 1. Socio-demographic characteristics, questions about the COVID-19 pandemic and to distance learning according to prevalence of depression, anxiety and stress, at different severities, during distance learning in times of covid-19 pandemic

\begin{tabular}{|c|c|c|c|c|c|c|c|c|c|c|c|c|c|c|c|c|c|c|}
\hline \multirow[b]{2}{*}{ Variables } & \multicolumn{5}{|c|}{ Depression, n (\%) } & \multirow[b]{2}{*}{ 产 } & \multicolumn{5}{|c|}{ Anxiety, n (\%) } & \multirow[b]{2}{*}{ 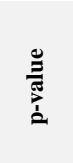 } & \multicolumn{5}{|c|}{ Stress, $\mathbf{n}(\%)$} & \multirow[b]{2}{*}{ לّ } \\
\hline & 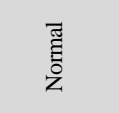 & $\frac{\bar{z}}{\bar{z}}$ & 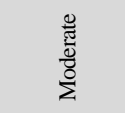 & 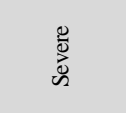 & 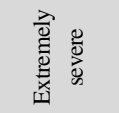 & & $\begin{array}{l}\overline{\widetilde{Z}} \\
\overline{\tilde{O}} \\
\mathrm{Z}\end{array}$ & $\frac{\bar{\pi}}{\bar{\Sigma}}$ & $\begin{array}{l}\frac{\pi}{\pi} \\
\frac{\pi}{0} \\
\sum \\
\sum\end{array}$ & 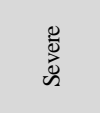 & 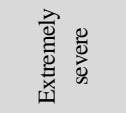 & & 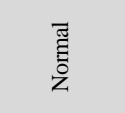 & $\frac{\pi}{\bar{z}}$ & $\begin{array}{l}\frac{0}{\pi} \\
\frac{\pi}{0} \\
\frac{0}{2}\end{array}$ & 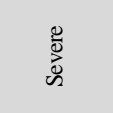 & 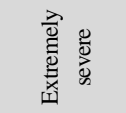 & \\
\hline \multicolumn{19}{|l|}{ Age (years) } \\
\hline $17-25$ & $27(22.5)$ & $8(6.7)$ & $13(10.8)$ & $15(12.5)$ & $22(18.3)$ & \multirow{3}{*}{0.67} & $23(19.2)$ & $7(5.8)$ & $9(7.5)$ & $4(3.3)$ & $42(35.0)$ & \multirow{3}{*}{0.31} & $25(20.8)$ & $14(11.7)$ & $12(10.0)$ & $11(9.2)$ & $23(19.2)$ & \multirow{3}{*}{0.16} \\
\hline $26-35$ & $13(10.8)$ & $2(1.7)$ & $7(5.8)$ & $3(2.5)$ & $6(5.0)$ & & $13(10.8)$ & $1(0.8)$ & $2(1.7)$ & $1(0.8)$ & $14(11.7)$ & & $18(15.0)$ & $2(1.7)$ & $4(3.3)$ & $1(0.8)$ & $6(5.0)$ & \\
\hline $35+$ & $3(2.5)$ & - & $1(0.8)$ & - & - & & $3(2.5)$ & - & $1(0.8)$ & - & - & & $3(2.5)$ & - & - & - & $1(0.8)$ & \\
\hline \multicolumn{19}{|l|}{ Gender } \\
\hline Male & $13(10.8)$ & & $9(7.5)$ & $6(5.0)$ & $1(0.8)$ & \multirow{2}{*}{0.00} & $13(10.8)$ & & $6(5.0)$ & & $10(8.3)$ & \multirow{2}{*}{0.02} & $21(17.5)$ & $1(0.8)$ & $5(4.2)$ & & $2(1.7)$ & \multirow{2}{*}{0.00} \\
\hline Female & $30(25.0)$ & $10(8.3)$ & $12(10.0)$ & $12(10.0)$ & $27(22.5)$ & & $26(21.7)$ & $8(6.7)$ & $6(5.0)$ & $5(4.2)$ & $46(38.3)$ & & $25(20.8)$ & $15(12.5)$ & $11(9.2)$ & $12(10.0)$ & $28(23.3)$ & \\
\hline Family incom & & & & & & & & & & & & & & & & & & \\
\hline $1-2$ & $10(8.3)$ & $1(0.8)$ & $1(0.8)$ & $3(2.5)$ & $5(4.2)$ & & $6(5.0)$ & $4(3.3)$ & - & $2(1.7)$ & $8(6.7)$ & & $4(3.3)$ & $7(5.8)$ & $1(0.8)$ & $5(4.2)$ & $3(2.5)$ & \\
\hline $3-4$ & $17(14.2)$ & $4(3.3)$ & $12(10.0)$ & $7(5.8)$ & $13(10.8)$ & 079 & $18(15.0)$ & $1(0.8)$ & $5(4.2)$ & $3(2.5)$ & $26(21.7)$ & & $20(16.7)$ & $8(6.7)$ & $7(5.8)$ & $2(1.7)$ & $16(13.3)$ & \\
\hline $5-6$ & $11(9.2)$ & $5(4.2)$ & $7(5.8)$ & $7(5.8)$ & $7(5.8)$ & 0.79 & $12(10.0)$ & $3(2.5)$ & $5(4.2)$ & - & $17(14.2)$ & 0.22 & $18(15.0)$ & - & $6(5.0)$ & $5(4.2)$ & $8(6.7)$ & 0.10 \\
\hline $6+$ & $5(4.2)$ & - & $1(0.8)$ & $1(0.8)$ & $3(2.5)$ & & $3(2.5)$ & - & $2(1.7)$ & - & $5(4.2)$ & & $4(3.3)$ & $1(0.8)$ & $2(1.7)$ & - & $3(2.5)$ & \\
\hline Marital statu: & & & & & & & & & & & & & & & & & & \\
\hline Single & $34(28.3)$ & $8(6.7)$ & $19(15.8)$ & $15(12.5)$ & $26(21.7)$ & & $30(25.0)$ & $7(5.8)$ & $9(7.5)$ & $4(3.3)$ & $52(43.3)$ & & $36(20.0)$ & $14(11.7)$ & $14(11.7)$ & $10(8.3)$ & $28(23.3)$ & \\
\hline Married & $8(6.7)$ & & $2(1.7)$ & $2(1.7)$ & $2(1.7)$ & & $7(5.8)$ & & $3(2.5)$ & $1(0.8)$ & $3(2.5)$ & & $9(7.5)$ & & $1(0.8)$ & $2(1.7)$ & $2(1.7)$ & \\
\hline Divorced & & $1(0.8)$ & - & - & $2(1 . .1)$ & 0.23 & $1(0.8)$ & & - & & & 0.14 & - & $1(0.8)$ & $1(0.0)$ & & - & 0.17 \\
\hline Stable union & $1(0.8)$ & $1(0.8)$ & - & $1(0.8)$ & - & & $1(0.8)$ & $1(0.8)$ & - & - & $1(0.8)$ & & $1(0.8)$ & $1(0.8)$ & $1(0.8)$ & - & - & \\
\hline Class shift & & & & & & & & & & & & & & & & & & \\
\hline Nightly & $17(14.2)$ & $2(1.7)$ & $6(5.0)$ & $8(6.7)$ & $5(4.2)$ & & $13(10.8)$ & $2(1.7)$ & $2(1.7)$ & $1(0.8)$ & $20(16.7)$ & & $19(15.8)$ & $4(3.3)$ & $8(6.7)$ & $2(1.7)$ & $5(4.2)$ & \\
\hline Full time & $26(21.7)$ & $8(6.7)$ & $15(12.5)$ & $10(8.3)$ & $23(19.2)$ & 0.22 & $26(21.7)$ & $6(5.0)$ & $10(8.3)$ & $4(3.3)$ & $36(30.0)$ & 0.77 & $27(22.5)$ & $12(10.0)$ & $8(6.7)$ & $10(8.3)$ & $25(20.8)$ & 0.05 \\
\hline Employment & id activity & & & & & & & & & & & & & & & & & \\
\hline No & $28(23.3)$ & $8(6.7)$ & $14(11.7)$ & $13(10.8)$ & $22(18.3)$ & & $28(23.3)$ & $7(5.8)$ & $7(5.8)$ & $4(3.3)$ & $39(32.5)$ & & $28(23.3)$ & $13(10.8)$ & $10(8.3)$ & $11(9.2)$ & $23(19.2)$ & \\
\hline Yes & $15(12.5)$ & $2(1.7)$ & $7(5.8)$ & $5(4.2)$ & $6(5.0)$ & 0.75 & $11(9.2)$ & $1(0.8)$ & $5(4.2)$ & $1(0.8)$ & $17(14.2)$ & 0.74 & $18(15.0)$ & $3(2.5)$ & $6(5.0)$ & $1(0.8)$ & $7(5.8)$ & 0.17 \\
\hline Internet avail & & & & & & & & & & & & & & & & & & \\
\hline No & $2(1.7)$ & & & - & $1(0.8)$ & & & & - & - & $3(2.5)$ & & & $2(1.7)$ & - & & $1(0.8)$ & \\
\hline Yes & $41(34.2)$ & $10(8.3)$ & $21(17.5)$ & $18(15.0)$ & $27(22.5)$ & 1.00 & $39(32.5)$ & $8(6.7)$ & $12(10.0)$ & $5(4.2)$ & $53(44.2)$ & 0.63 & $46(38.3)$ & $14(11.7)$ & $16(13.3)$ & $12(10.0)$ & $29(24.2)$ & 0.09 \\
\hline Internet tools & vledge & & & & & & & & & & & & & & & & & \\
\hline No & $2(1.7)$ & - & $3(2.5)$ & $6(5.0)$ & $5(4.2)$ & & $2(1.7)$ & - & - & - & $14(11.7)$ & & $5(4.2)$ & $1(0.8)$ & $1(0.8)$ & $3(2.5)$ & $6(5.0)$ & \\
\hline Yes & $41(34.2)$ & $10(8.3)$ & $18(15.0)$ & $12(10.0)$ & $23(19.2)$ & 0.02 & $37(30.8)$ & $8(6.7)$ & $12(10.0)$ & $5(4.2)$ & $42(35.0)$ & 0.02 & $41(34.2)$ & $15(12.5)$ & $15(12.5)$ & $9(7.5)$ & $24(20.0)$ & 0.42 \\
\hline Satisfied with & ince learnin & & & & & & & & & & & & & & & & & \\
\hline No & $22(18.3)$ & $8(6.7)$ & $8(6.7)$ & $6(5.0)$ & $23(19.2)$ & & $21(17.5)$ & $7(5.8)$ & $5(4.2)$ & $2(1.7)$ & $32(26.7)$ & & $20(16.7)$ & $11(9.2)$ & $5(4.2)$ & $10(8.3)$ & $21(17.5)$ & \\
\hline Yes & $21(17.5)$ & $2(1.7)$ & $13(10.8)$ & $12(10.0)$ & $5(4.2)$ & 0.00 & $18(15.0)$ & $1(0.8)$ & $7(5.8)$ & $3(2.5)$ & $24(20.0)$ & 0.30 & $26(21.7)$ & $5(4.2)$ & $11(9.2)$ & $2(1.7)$ & $9(7.5)$ & 0.00 \\
\hline Had COVID- & & & & & & & & & & & & & & & & & & \\
\hline No & $39(32.5)$ & $9(7.5)$ & $19(15.8)$ & $18(15.0)$ & $22(18.3)$ & 024 & $34(28.3)$ & $8(6.7)$ & $11(9.2)$ & $5(4.2)$ & $49(40.8)$ & 0.95 & $40(33.3)$ & $15(12.5)$ & $16(13.3)$ & $12(10.0)$ & $24(20.0)$ & \\
\hline Yes & $4(3.3)$ & $1(0.8)$ & $2(1.7)$ & - & $6(5.0)$ & 0.24 & $5(4.2)$ & - & $1(0.8)$ & - & $7(5.8)$ & 0.95 & $6(5.0)$ & $1(0.8)$ & - & - & $6(5.0)$ & 0.20 \\
\hline Fear of contr & COVID-19 & & & & & & & & & & & & & & & & & \\
\hline No & $14(11.7)$ & & $9(7.5)$ & $1(0.8)$ & - & & $14(11.7)$ & & $5(4.2)$ & - & $5(4.2)$ & & $23(19.2)$ & & - & & $1(0.8)$ & \\
\hline Yes & $29(24.2)$ & $10(8.3)$ & $12(10.0)$ & 17 (14.2) & $28(23.3)$ & 0.00 & $25(20.8)$ & $8(6.7)$ & $7(5.8)$ & $5(4.2)$ & $51(42.5)$ & 0.00 & $23(19.2)$ & $16(13.3)$ & $16(13.3)$ & $12(10.0)$ & $29(24.2)$ & 0.00 \\
\hline A family men & had COVID & & & & & & & & & & & & & & & & & \\
\hline No & $30(25.0)$ & $6(5.0)$ & $3(2.5)$ & $7(5.8)$ & $5(4.2)$ & & $26(21.7)$ & $6(5.0)$ & $6(5.0)$ & $2(1.7)$ & $11(9.2)$ & & $18(15.0)$ & $12(10.0)$ & $8(6.7)$ & $8(6.7)$ & $5(4.2)$ & \\
\hline Yes & $13(10.8)$ & $4(3.3)$ & $18(15.0)$ & $11(9.2)$ & $23(19.2)$ & 0.00 & $13(10.8)$ & $2(1.7)$ & $6(5.0)$ & $3(2.5)$ & $45(37.5)$ & 0.00 & $28(23.3)$ & $4(3.3)$ & $8(6.7)$ & $4(3.3)$ & $25(20.8)$ & 0.00 \\
\hline A friend had & ID-19 & & & & & & & & & & & & & & & & & \\
\hline No & $27(22.5)$ & $6(5.0)$ & $5(4.2)$ & $5(4.2)$ & $3(2.5)$ & & $24(20.0)$ & $5(4.2)$ & $5(4.2)$ & $4(3.3)$ & $8(6.7)$ & & $19(15.8)$ & $9(7.5)$ & $5(4.2)$ & $7(5.8)$ & $6(5.0)$ & \\
\hline Yes & $16(13.3)$ & $4(3.3)$ & $16(13.3)$ & $13(10.8)$ & $25(20.8)$ & 0.00 & $15(12.5)$ & $3(2.5)$ & $7(5.8)$ & $1(0.8)$ & $48(40.0)$ & 0.00 & $27(22.5)$ & $7(5.8)$ & $11(9.2)$ & $5(4.2)$ & $24(20.0)$ & 0.05 \\
\hline In social deta & & & & & & & & & & & & & & & & & & \\
\hline No & $10(8.3)$ & - & $3(2.5)$ & $2(1.7)$ & $5(4.2)$ & & $7(5.8)$ & - & $1(0.8)$ & - & $12(10.0)$ & & $10(8.3)$ & $3(2.5)$ & $2(1.7)$ & $1(0.8)$ & $4(3.3)$ & \\
\hline Yes & $33(27.5)$ & $10(8.3)$ & $18(15.0)$ & $16(13.3)$ & $23(19.2)$ & 0.51 & $32(26.7)$ & $8(6.7)$ & $11(9.2)$ & $5(4.2)$ & 44 (36.7) & 0.58 & $36(30.0)$ & $13(10.8)$ & 14 (11.7) & $11(9.2)$ & $26(21.7)$ & 0.81 \\
\hline
\end{tabular}

* Fisher's exact test. 
Table 2. Bivariate logistic regression analysis of socio-demographic characteristics, questions about the COVID-19 pandemic and to distance learning of dentistry students with depression, anxiety and stress (low/high) during distance learning in times of COVID-19 pandemic

\begin{tabular}{|c|c|c|c|c|c|c|c|c|c|}
\hline \multirow{2}{*}{ Variables } & \multicolumn{3}{|c|}{ Depression } & \multicolumn{3}{|c|}{ Anxiety } & \multicolumn{3}{|c|}{ Stress } \\
\hline & $p$ & OR & $95 \% \mathrm{CI}$ & $p$ & OR & $95 \% \mathrm{CI}$ & $p$ & OR & $95 \%$ CI \\
\hline \multicolumn{10}{|l|}{ Age (years) } \\
\hline $17-25$ & 1 & & & 1 & & & 1 & & \\
\hline $26-35$ & 0.16 & 0.531 & $0.219-1.28$ & 0.58 & 0.795 & $0.34-1.81$ & 0.08 & 0.43 & $0.17-1.12$ \\
\hline $35+$ & 0.99 & 0.00 & $0.00-0.00$ & 0.99 & 0.00 & $0.00-0.00$ & 0.55 & 0.50 & $0.05-5.00$ \\
\hline \multicolumn{10}{|l|}{ Gender } \\
\hline Male & 1 & & & 1 & & & 1 & & \\
\hline Female & 0.76 & 2.35 & $0.91-6.07$ & 0.04 & 2.42 & $1.01-5.78$ & 0.00 & 10.58 & $2.37-47.20$ \\
\hline \multicolumn{10}{|c|}{ Family income } \\
\hline $1-2$ & 1 & & & 1 & & & 1 & & \\
\hline $3-4$ & 0.85 & 0.90 & $0.31-2.60$ & 0.71 & 1.20 & $0.43-3.38$ & 0.63 & 0.77 & $0.26-2.22$ \\
\hline $5-6$ & 0.87 & 0.91 & $0.30-2.78$ & 0.77 & 0.85 & $0.28-2.52$ & 0.71 & 0.81 & $0.26-2.49$ \\
\hline $6+$ & 1.00 & 1.00 & $0.21-4.70$ & 1.00 & 1.00 & $0.21-4.56$ & 0.59 & 0.64 & $0.12-3.25$ \\
\hline \multicolumn{10}{|c|}{ Marital status } \\
\hline Single & 1 & & & 1 & & & 1 & & \\
\hline Married & 0.40 & 0.59 & $0.17-2.02$ & 0.07 & 0.32 & $0.09-1.11$ & 0.52 & & $0.19-2.29$ \\
\hline Divorced & 1.00 & 0.00 & $0.00-0.00$ & 1.00 & 0.00 & $0.00-0.00$ & 1.00 & 0.00 & $0.00-0.00$ \\
\hline Stable union & 0.81 & 0.74 & $0.06-8.47$ & 0.47 & 0.41 & $0.03-4.67$ & 0.99 & 0.00 & $0.00-0.00$ \\
\hline \multicolumn{10}{|l|}{ Class shift } \\
\hline Full time & 1 & & & 1 & & & 1 & & \\
\hline Nightly & 0.52 & 0.77 & $0.34-1.72$ & 0.50 & 1.29 & $0.59-2.80$ & 0.01 & 0.30 & $0.12-0.76$ \\
\hline \multicolumn{10}{|c|}{ Employment or paid activity } \\
\hline No & 1 & & & 1 & & & 1 & & \\
\hline Yes & 0.32 & 0.65 & $0.28-1.50$ & 0.93 & 1.03 & $0.47-2.27$ & 0.7 & 0.44 & $0.18-1.09$ \\
\hline \multicolumn{10}{|c|}{ Internet available } \\
\hline No & 1 & & & 1 & & & 1 & & \\
\hline Yes & 0.85 & 1.25 & $0.11-14.18$ & 0.99 & 0.00 & $0.00-0.00$ & 0.95 & 1.07 & $0.09-12.25$ \\
\hline \multicolumn{10}{|c|}{ Internet tools knowledge } \\
\hline No & 1 & & & 1 & & & 1 & & \\
\hline Yes & 0.01 & 0.23 & $0.07-0.71$ & 0.00 & 0.11 & $0.02-0.54$ & 0.06 & 0.36 & $0.12-1.05$ \\
\hline \multicolumn{10}{|c|}{ Satisfied with distance learning } \\
\hline No & 1 & & & 1 & & & 1 & & \\
\hline Yes & 0.21 & 0.61 & $0.29-1.31$ & 0.98 & 1.00 & $0.49-2.07$ & 0.00 & 0.30 & $0.13-0.69$ \\
\hline \multicolumn{10}{|c|}{ Had COVID-19 } \\
\hline No & 1 & & & 1 & & & 1 & & \\
\hline Yes & 0.54 & 1.43 & $0.45-4.5$ & 0.81 & 1.14 & $0.36-3.63$ & 0.37 & 1.69 & $0.52-5.40$ \\
\hline \multicolumn{10}{|c|}{ Fear of contracting COVID-19 } \\
\hline No & 1 & & & 1 & & & 1 & & \\
\hline Yes & 0.00 & 20.29 & $2.63-153.36$ & 0.00 & 5.32 & $1.83-15.43$ & 0.00 & 17.14 & $2.22-132.19$ \\
\hline \multicolumn{10}{|c|}{ A family member had COVID-19 } \\
\hline No & 1 & & & 1 & & & 1 & & \\
\hline Yes & 0.00 & 3.15 & $1.41-7.03$ & 0.00 & 6.68 & $2.96-15.05$ & 0.06 & 2.11 & $0.96-4.67$ \\
\hline \multicolumn{10}{|c|}{ A friend had COVID-19 } \\
\hline No & 1 & & & 1 & & & 1 & & \\
\hline Yes & 0.00 & 5.01 & $2.06-12.18$ & 0.00 & 5.55 & $2.45-12.55$ & 0.22 & 1.63 & $0.74-3.61$ \\
\hline \multicolumn{10}{|c|}{ In social detachment } \\
\hline No & 1 & & & 1 & & & 1 & & \\
\hline Yes & 0.73 & 1.18 & $0.43-3.23$ & 0.37 & 0.64 & $0.24-1.70$ & 0.30 & 1.76 & $0.59-5.24$ \\
\hline
\end{tabular}

COVID-19 and who had a familiar or a friend diagnosed with COVID-19 presented more anxiety or stress. 
Dental education is competitive and rigorous, placing emotional, psychological, as well as physical demands on students, from the first year of the course ${ }^{28}$. The prevalence of depression, anxiety and stress among dental students analyzed in this study were high: $55.9 \%$, $60.8 \%$ and $48.4 \%$ of students had moderate or above levels of depression, anxiety and stress, respectively. Scores of moderate severity and above indicate a possible problem requiring intervention $^{23,29}$. These results reinforce the results of the majority of studies that also found alarming levels of depression, anxiety and stress among dental students, using the DASS-21, even before the COVID-19 pandemic po,31,32, although $^{30}$ the study developed by Jowkar et al. $(2020)^{33}$ has shown that the dental students at Shiraz School of Dentistry (Iran) experienced normal levels of psychological distress. Recently, Hakami et al. $(2020)^{34}$ evaluated the psychological impact (DASS-21) of the COVID-19 pandemic on dental students in Saudi Arabia and also found a similar prevalence of depression, anxiety and stress, $60.6 \%, 37 \%$ and $34.9 \%$, respectively. Another study evaluated the prevalence of depression (Patient Health Questionnaire - PHQ9) during the COVID-19 lockdown among dental students of India and found that ony $12 \%$ had no depression, whereas the proportions of students with mild, moderate, and severe depression were $34 \%, 27 \%$, and $27 \%$, respectively ${ }^{35}$.

Certain degrees of stress improve performance and prevent boredom, but the persistence of chronic stressors or adverse experiences of stress-related symptoms increase anxiety and depression levels ${ }^{16}$. Given our results and those presented by studies carried out before and after the COVID-19 pandemic, dental students are subject to develop alarming levels of depression, anxiety and stress. This can be explained by the fact that during their dental studies, students must learn clinical skills in addition to managing a busy and challenging academic curriculum ${ }^{15}$. Sources of stress in preclinical years usually stem from academic factors such as workload and grades, whereas in clinical years, students experience additional stress in learning clinical procedures and dealing with difficult patients ${ }^{15}$.

Considering the current pandemic scenario where the dental practice is restricted due to the risk of exposure to COVID-19, dental students are experiencing tremendous pressure, which is affecting their mental health ${ }^{34}$. The result is increased fear and psychological distress among dental students ${ }^{34}$. According to Chakraborty et al. $(2020)^{35}$, during the COVID-19 lockdown, the depression among dental students is associated with worry about professional growth due to reduced clinical training, especially among interns, the possibility of the increased course completion length and employment prospects ${ }^{35}$. Our results showed that female students presented more chances of developing a high level of depression, anxiety or stress, reinforcing the recent findings of Hakami et al. (2020) ${ }^{34}$. These results were also similar to those studies reporting on the mental health status of dental students in several other countries, even before

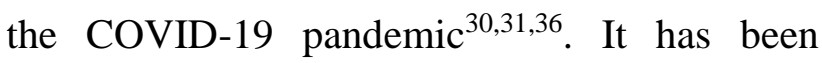
reported that clinical factors, such as technical skills, are the most stressful for female students ${ }^{37}$. Some studies, including several with college student populations, identify women as being at greater risk of psychological distress during the COVID-19 pandemic ${ }^{38,39,40}$. This observation may be attributable to higher levels of preexisting psychopathology in women as well as gender differences in fear processing, which could translate to exacerbations of symptoms ${ }^{41}$. Also, male students tend to have higher confidence in the computer skills necessary for the transition to online course delivery ${ }^{38}$. Meanwhile, women are more concerned about 
impacts on their professional career and ability to study than men, on average ${ }^{38}$.

An interesting result found in this study was that students who were fear of contracting COVID-19 presented more chances of developing a high level of depression, anxiety or stress. Chakraborty et al. $(2020)^{35}$ also observed similar results among undergraduate dental students. These authors showed that the fear of contracting COVID-19 among students was positively related to depression scores. Psychological implications such as fear and anxiety are natural in pandemics, especially when the number of infected individuals and mortality rates is increasing sharply ${ }^{42}$. The repercussions of the rapid spread of COVID-19, which has affected millions of people worldwide, ranging from being isolated and quarantined to fatality have resulted in considerable psychological stress and fear ${ }^{43}$. With the prolonged incubation period of the coronavirus (as long as 14 days), it is virtually impossible to pinpoint an individual's exposure to the virus ${ }^{43}$. In addition, there was no vaccine at the time of research or approved treatment, which further enhances anxiety and fear upon the thought of getting infected. It is important to highlight that for some individuals the anxiety in response to a pandemic threat can become excessive and maladaptive. Excessive anxiety can be debilitating and lead to severe impairment to mental health ${ }^{44}$.

In this study, it was observed that dental students who had a familiar or a friend diagnosed with COVID-19 also presented more chances of developing a high level of depression, anxiety or stress. This finding is compatible with another study, where university students who knew someone infected with COVID-19 experienced higher levels of psychological impact ${ }^{34}$. The finding that knowing someone infected is a risk factor for psychological impacts of COVID-19 is intuitive $^{45}$. Familiarity can increase the salience and perceived risk of becoming infected and dealing with subsequent health concerns, like COVID-19-related death ${ }^{45}$.

University administrators could best serve students if they better understand the impacts of COVID-19 and the risk factors of its psychological impacts ${ }^{46}$. These impacts are of critical importance to warrant immediate mental health interventions focused on prevention and treatment. Several strategies for stress management among dental students have been introduced and discussed in the literature, including relaxation strategies, interpersonal approaches such as counseling systems, programs designed to improve studying and testtaking skills and stress management workshops ${ }^{47}$. Because stress in the dental educational environment is typically unavoidable, stress management strategies can be recommended as an early and integral part of the dental curriculum ${ }^{31}$. These strategies could focus mainly on improving the perception of stressful situations, the development of coping skills and the avoidance of maladaptive coping ${ }^{31}$. Additionally, more emphasis should be placed on the importance of humanistic faculty-student relationships. It is relevant to highlight that the psychiatric and counseling services have historically been underutilized by college students ${ }^{48}$. Thus, in this pandemic moment, understanding what subpopulations among dental students may suffer from unique combinations of psychological impacts may facilitate targeted interventions and successful treatment and coping strategies for individuals at greatest risk.

The findings of this study are of great importance because it is the first study that evaluated the impact of the COVID-19 pandemic on the mental health of dental students in Brazil. Although this study has several strengths, such as 
the excellent response rate, probabilistic sampling method, and the simple, concise and validated test tool, it also has some limitations. Limitations to this study include its crosssectional design, which precludes causal inferences, and was an online survey, so there was a possibility of reporting bias. Besides that, only one dental school was evaluated in the present study. Lastly, our measures were retrospective rather than longitudinal, which decreases our ability to say with confidence that the reported impacts were caused by COVID- 19 . Further investigations on greater populations of dental students including the students of public and private dental schools in other dental institutions need to be done as well.

\section{CONCLUSION}

This study found high levels of depression, anxiety and stress among dental students during the COVID-19 pandemic and distance learning. Moreover, female students, students who were fear of contracting COVID-19 and who had a familiar or a friend diagnosed with COVID-19 presented more chances of developing a high level of depression, anxiety or stress. This study suggests that mental health from dental students should be carefully monitored during the COVID-19 pandemic and that universities should provide psychological services-oriented and adapted to mitigate the mental impact on students.

\section{RESUMO}

Depressão, ansiedade e estresse entre estudantes de Odontologia durante a pandemia da COVID-19 e o ensino remoto

Este estudo teve como objetivo mensurar os níveis de depressão, ansiedade e estresse entre estudantes de Odontologia durante a pandemia da COVID-19 e o ensino à distância. Esta pesquisa transversal, realizada por meio de um questionário on-line, foi realizada em uma
Faculdade de Odontologia do Brasil, entre julho e agosto de 2020. Os participantes foram avaliados por meio da versão adaptada e validada do questionário Escala de Depressão, Ansiedade e Estresse (DASS-21). Para análise dos dados foram realizados o Teste Exato de Fisher e a Análise de Regressão Logística Bivariada, por meio do software SPSS. Participaram do estudo 120 estudantes de odontologia (taxa de resposta de $87.60 \%$ ). Destes, $75.8 \%$ deles eram do sexo feminino e a média de idade foi de 23,35 anos ( \pm $6,07)$. A maioria dos alunos era solteira $(85,0 \%)$, estudava em tempo integral $(68,3 \%)$ e não tinha vínculo empregatício (70,8\%). Algum nível de depressão, ansiedade e estresse foi observado em $64,2 \%, \quad 67,5 \%$ e $61,7 \%$ dos alunos, respectivamente. As gravidades da depressão, da ansiedade e do estresse estiveram significativamente associadas $(\mathrm{p}<0.05)$ ao sexo do aluno, ao medo de contrair a COVID-19 e à condição de ter um membro da família que já teve a COVID-19. Estudantes do sexo feminino, estudantes com medo de contrair a COVID-19 e que tiveram um familiar ou amigo com diagnóstico de COVID-19 apresentaram maiores chances de desenvolver um alto nível de depressão, ansiedade ou estresse. Concluímos que os estudantes de odontologia apresentaram altos níveis de depressão, ansiedade e estresse durante a pandemia da COVID-19 e o ensino à distância. Este estudo sugere que a saúde mental dos estudantes de odontologia deve ser monitorada cuidadosamente durante a pandemia da COVID-19.

Descritores: Infecção por SARS-CoV-2. Educação em Odontologia. Saúde Mental.

\section{REFERENCES}

1. Mahase E. China coronavirus: WHO declares international emergency as death toll exceeds 200. BMJ. 2020;368:m408.

2. Burki T. COVID-19 in Latin America. The Lancet. 2020;5(1):547-548.

3. Lobo AA, Cardoso-Dos-Santos AC, Rocha MS et al. COVID-19 epidemic in Brazil: Where are we at? Int $J$ Infect Dis. 2020;97:382-5. 
4. Xiong J, Lipsitz O, Nasri F et al. Impact of COVID-19 pandemic on mental health in the general population: A systematic review. J Affect Disord. 2020;277:55-64.

5. Zhai $Y, D u X$. Mental health care for international Chinese students affected by the COVID-19 outbreak. Lancet Psychiatry. 2020;7(4):e22.

6. Araújo FJO, de Lima LSA, Cidade PIM et al. Impact of Sars-Cov-2 and its reverberation in global higher education and mental health. Psychiatry Res. 2020;288:112977.

7. Sahu P. Closure of universities due to coronavirus disease 2019 (COVID-19): impact on education and mental health of students and academic staff. Cureus. 2020;12:e7541.

8. Zivin K, Eisenberg D, Gollust SE, Golberstein E. Persistence of mental health problems and needs in a college student population. J Affect Disord. 2009;117:1805.

9. Beiter R, Nash R, McCrady M et al. The prevalence and correlates of depression, anxiety, and stress in a sample of college students. J Affect Disord. 2015;173:90-6.

10. Rith-Najarian LR, Boustani MM, Chorpita BF. A systematic review of prevention programs targeting depression, anxiety, and stress in university students. J Affect Disord. 2019;257:568-84.

11. Kecojevic A, Basch CH, Sullivan M, Davi NK. The impact of the COVID-19 epidemic on mental health of undergraduate students in New Jersey, cross-sectional study. PLoS One. 2020;15(9):e0239696.

12. Chi X, Becker B, Yu Q et al. Prevalence and Psychosocial Correlates of Mental Health Outcomes Among Chinese College Students During the Coronavirus Disease (COVID19) Pandemic. Front Psychiatry.
2020;11:803.

13. Odriozola-González P, Planchuelo-Gómez A, Irurtia MJ, Luis-García R. Psychological effects of the COVID-19 outbreak and lockdown among students and workers of a Spanish university. Psychiatry Res. 2020;290:113108.

14. Piazza-Waggoner CA, Cohen LL, Kohli K, Taylor BK. Stress management for dental students performing their first pediatric restorative procedure. $\mathrm{J}$ Dent Edu. 2003;67(5):542-8.

15. Elani HW, Allison PJ, Kumar RA, Mancini $\mathrm{L}$ et al. A systematic review of stress in dental students. J Dent Educ. 2014;78(2):226-42.

16. Alzahem AM, Van der Molen HT, Alaujan $\mathrm{AH}$ et al. Stress amongst dental students: a systematic review. Eur J Dent Educ. 2011;15(1):8-18.

17. Goldstein MB. Interpersonal support and coping among first-year dental students. J Dent Edu. 1980;44(4):202-5.

18. Morse Z, Dravo U. Stress levels of dental students at the Fiji School of Medicine. Eur J Dent Edu. 2007;11(2):99-103.

19. Iyer P, Aziz K, Ojcius DM. Impact of COVID-19 on dental education in the United States. J Dent Educ. 2020;84(6):718-22.

20. Desai BK. Clinical implications of the COVID-19 pandemic on dental education. J Dent Educ. 2020;84(5):512.

21. Webster J, Hackley P. Teaching effectiveness in technology mediated distance learning. Acad Manage J. 1997;40:1282-1309.

22. Grimshaw J. SURGE (The SUrvey Reporting GuidelinE). In: Moher D, Altman DG, Schulz K et al. Guidelines for reporting health research: A user's manual. $1^{\text {st }} \mathrm{ed}$. Hoboken (NJ): John Wiley \& Sons, 2014. p.206-13. 
23. Lovibond PF, Lovibond SH. The structure of negative emotional states: comparison of the Depression Anxiety Stress Scales (DASS) with the Beck depression and anxiety inventories. Behav Res Ther. 1995;33(3):335-43.

24. Lovibond SH, Lovibond PF. Manual for the Depression Anxiety Stress Scales. $4^{\text {th }}$ ed. Psychology Foundation: Sydney, 2004.

25. Gloster AT, Rhoades HM, Novy D et al. Psychometric properties of the Depression Anxiety and Stress Scale-21 in older primary care patients. J Affect Disord. 2008;110(3):248-59.

26. Henry JD, Crawford JR. The short-form version of the Depression Anxiety Stress Scales (DASS-21): construct validity and normative data in a large non-clinical sample. Br J Clin Psychol. 2005;44(2):22739.

27. Vignola RCB, Tucci AM. Adaptation and validation of the depression, anxiety and stress scale (DASS) to Brazilian Portuguese. J Affect Disord. 2014;155:104-9.

28. Burk DT, Bender DJ. Use and perceived effectiveness of student support services in a first-year dental student population. J Dent Educ. 2005;69(10):1148-60.

29. Crawford JR, Henry JD. The Depression Anxiety Stress Scales (DASS): normative data and latent structure in a large nonclinical sample. $\mathrm{Br} \mathrm{J}$ Clin Psychol. 2003;42(2):111-31.

30. Aboalshamat K, Hou X, Strodl E. Psychological wellbeing status among medical and dental students in Makkah, Saudi Arabia: A cross-sectional study. Medical Teacher. 2015;37(sup1):S75-81.

31. Basudan S, Binanzan N, Alhassan A. Depression, anxiety and stress in dental students. Int J Med Educ. 2017;24(8):17986.
32. Stormon N, Ford PJ, Kisely $\mathrm{S}$ et al. Depression, anxiety and stress in a cohort of Australian dentistry students. Eur J Dent Educ. 2019;23(4):507-14.

33. Jowkar Z, Masoumi M, Mahmoodian H. Psychological Stress and Stressors Among Clinical Dental Students at Shiraz School of Dentistry. Iran. Adv Med Educ Pract. 2020;1:113-20.

34. Hakami Z, Khanagar SB, Vishwanathaiah S et al. Psychological impact of the coronavirus disease 2019 (COVID-19) pandemic on dental students: A nationwide study. J Dent Educ. 2020:1-10.

35. Chakraborty T, Subbiah GK, Damade Y. Psychological Distress during COVID-19 Lockdown among Dental Students and Practitioners in India: A Cross-Sectional Survey. Eur J Dent. 2020;14(supl 1):S70-8.

36. Moore R, Madsen, LV, Trans M. Stress sensitivity and signs of anxiety or depression among first year clinical dental and medical students. Open J Med Psychol. 2020; 9:7-20.

37. Al Sowygh ZH, Alfadley AA, Al-Saif MI, Al-Wadei SH. Perceived causes of stress among Saudi dental students. King Saud University J Dent Sci. 2013;4(1):7-15.

38. Aristovnik A, Keržič D, Ravšelj D, Tomaz evič N, Umek L. Impacts of the COVID-19 pandemic on life of higher education students: A global perspective. Sustainability. 2020;12:8438.

39. Wang C, Zhao H. The impact of COVID-19 on anxiety in Chinese university students. Front Psychol. 2020;11:1168.

40. Wenham C, Smith J, Morgan R. The Gender and COVID-19 Working Group. COVID19: The gendered impacts of the outbreak. Lancet. 2020;395:846-8.

41. Felmingham K, Williams LM, Kemp AH et al. Neural responses to masked fear faces: Sex differences and trauma exposure in 
posttraumatic stress disorder. $\mathrm{J}$ of Abnorm Psychol. 2010;119:241-7.

42. Ahmed MA, Jouhar R, Ahmed N. Fear and Practice Modifications among Dentists to Combat Novel Coronavirus Disease (COVID-19) Outbreak. Int J Environ Res Public Health. 2020;17(8):2821.

43. Moorthy V, Restrepo AMH, Preziosi MP, Swaminathan S. Active quarantine measures are the primary means to reduce the fatality rate of COVID-19. Bull. World Health Organ. 2020;98:150.

44. Asmundson JGG, Taylor S. How health anxiety influences responses to viral outbreaks like COVID-19: what all decisionmakers, health authorities, and health care professionals need to know. J. Anxiety Disord. 2020;71:102211.
45. Bitan DT, Grossman-Giron A, Bloch Y et al. Fear of COVID-19 scale: Psychometric characteristics, reliability and validity in the Israeli population. Psychiatry Research. 2020;289:113100.

46. Browning MHEM, Larson LR, Sharaievska I et al. Psychological impacts from COVID19 among university students: Risk factors across seven states in the United States. PLoS ONE. 2021;16(1):e0245327.

47. Alzahem AM, Van der Molen HT, Alaujan AH, De Boer BJ. Stress management in dental students: a systematic review. Adv Med Educ Pract. 2014;5:167-76.

48. Hunt J, Eisenberg D. Mental health problems and help-seeking behavior among college students. J Adolesc Health. 2010;46:3-10.

\section{Correspondence to:}

Dr. Francisco Ivison Rodrigues Limeira e-mail: ivisonodontoce@ hotmail.com Rua Engenheiro Célso Murta, 600 Olga Correa 39803-087 Teófilo Otoni/MG Brazil 